\title{
Article \\ Advanced Current Collectors with Carbon Nanofoams for Electrochemically Stable Lithium-Sulfur Cells
}

\author{
Shu-Yu Chen ${ }^{1}$ and Sheng-Heng Chung ${ }^{1,2, *}$ \\ 1 Department of Materials Science and Engineering, National Cheng Kung University, No. 1, \\ University Road, Tainan City 701, Taiwan; f64071203@gs.ncku.edu.tw \\ 2 Hierarchical Green-Energy Materials Research Center, National Cheng Kung University, No. 1, \\ University Road, Tainan City 701, Taiwan \\ * Correspondence: SHChung@gs.ncku.edu.tw
}

check for updates

Citation: Chen, S.-Y.; Chung, S.-H. Advanced Current Collectors with Carbon Nanofoams for Electrochemically Stable Lithium-Sulfur Cells. Nanomaterials 2021, 11, 2083. https://doi.org/ 10.3390/nano11082083

Academic Editor: Young-Si Jun

Received: 26 July 2021

Accepted: 14 August 2021

Published: 17 August 2021

Publisher's Note: MDPI stays neutral with regard to jurisdictional claims in published maps and institutional affiliations.

Copyright: (C) 2021 by the authors. Licensee MDPI, Basel, Switzerland. This article is an open access article distributed under the terms and conditions of the Creative Commons Attribution (CC BY) license (https:/ / creativecommons.org/licenses/by/ $4.0 /)$.

\begin{abstract}
An inexpensive sulfur cathode with the highest possible charge storage capacity is attractive for the design of lithium-ion batteries with a high energy density and low cost. To promote existing lithium-sulfur battery technologies in the current energy storage market, it is critical to increase the electrochemical stability of the conversion-type sulfur cathode. Here, we present the adoption of a carbon nanofoam as an advanced current collector for the lithium-sulfur battery cathode. The carbon nanofoam has a conductive and tortuous network, which improves the conductivity of the sulfur cathode and reduces the loss of active material. The carbon nanofoam cathode thus enables the development of a high-loading sulfur cathode $\left(4.8 \mathrm{mg} \mathrm{cm}^{-2}\right)$ with a high discharge capacity that approaches $500 \mathrm{~mA} \cdot \mathrm{h} \mathrm{g}^{-1}$ at the $\mathrm{C} / 10$ rate and an excellent cycle stability that achieves $90 \%$ capacity retention over 100 cycles. After adopting such an optimal cathode configuration, we superficially coat the carbon nanofoam with graphene and molybdenum disulfide $\left(\mathrm{MoS}_{2}\right)$ to amplify the fast charge transfer and strong polysulfide-trapping capabilities, respectively. The highest charge storage capacity realized by the graphene-coated carbon nanofoam is $672 \mathrm{~mA} \cdot \mathrm{h} \mathrm{g}^{-1}$ at the $\mathrm{C} / 10 \mathrm{rate}$. The $\mathrm{MoS}_{2}$-coated carbon nanofoam features high electrochemical utilization attaining the high discharge capacity of $633 \mathrm{~mA} \cdot \mathrm{h} \mathrm{g}^{-1}$ at the $\mathrm{C} / 10$ rate and stable cyclability featuring a capacity retention approaching $90 \%$.
\end{abstract}

Keywords: lithium-sulfur batteries; sulfur cathode; carbon nanofoam; graphene; $\mathrm{MoS}_{2}$

\section{Introduction}

The increasing demand for an advanced energy storage system has attracted the attention of researchers and inventors, who have investigated the possibility of next-generation rechargeable batteries with a high energy density and low cost [1-3]. Manganese (or nickel)-rich lithium nickel manganese cobalt oxide cathodes and sulfur cathodes are the most promising candidates, with sulfur having the highest charge storage capacity of $1672 \mathrm{~mA} \cdot \mathrm{h} \mathrm{g}^{-1}$ and the lowest cost among all solid-state cathode materials [4-6]. Moreover, electrochemical conversion of the sulfur cathode occurs reversibly at a safe operating voltage of $\sim 2.1 \mathrm{~V}$ and, most importantly, bypasses the limitation imposed by the crystalline structure of commercial insertion-type lithium-ion battery cathodes [5-8]. However, the conversion battery chemistry of lithium-sulfur batteries involves the repeated interconversion of the active material between solid and liquid states, along with the formation of new sulfur-based compounds [5,9-11]. At the full charge and discharge stages, the insulating sulfur and sulfides are deposited at the sulfur cathode. The resulting high cathode resistance makes it difficult for a sulfur cathode to effectively and reversibly utilize its high charge storage capacity [9-11]. During the discharge/charge processes, solid-state sulfur and sulfides convert to liquid-state lithium polysulfides $\left(\mathrm{Li}_{2} \mathrm{~S}_{x}, 4 \leq x \leq 8\right)$. The polysulfides are highly soluble in ether-based liquid electrolytes. Upon dissolution, the polysulfides irreversibly diffuse out of the cathode and uncontrollably migrate across the 
whole cell, which deteriorates both the electrodes and the electrolyte. Such polysulfide diffusion causes the capacity to fade quickly, resulting in a short cycle life [11-13].

To address these scientific issues, mainstream lithium-sulfur technologies are currently focused on optimizing the sulfur cathode [14-16] by the addition of conductive additives for high electrochemical utilization [14-23] and by the inclusion of porous substrates for strong polysulfide stabilization [14-16,20-25]. One of the most promising methods is the synthesis of sulfur-based nanocomposites that have various conductive and porous substrates and can easily form composites with sulfur. The resulting sulfur-based nanocomposite is subsequently coated onto an aluminum foil current collector by using additional carbon black and binder, followed by extra cathode preparation steps to further optimize cathode conductivity and integrity [14-16]. However, sulfur cathode chemistry dictates that the polysulfides that form during the intermediate charge and discharge stages inevitably diffuse out of the flat aluminum foil, eventually causing the loss of active material and damaging the integrity of the cathode. Moreover, the redeposition of the diffusing polysulfides in due course covers the two-dimensional cathode and hastens cathode failure [10-12]. To avoid this effect, the use of a porous current collector in the sulfur cathode has been proposed. A porous current collector is usually a conductive porous substrate with a conductive network that can transfer electrons throughout the cathode region [12-14]. Its porous structure ensures good electrolyte wetting and penetration, hosts the active material, and slows down the diffusion of polysulfides [26-29].

Here, we present a carbon nanofoam substrate as a porous current collector that features a carbon nanofiber skeleton with attached nanoporous carbon clusters. This conductive and porous carbon nanofoam substrate enables high electrochemical utilization and stability of the large amount of hosted sulfur [12-14,26-28]. The carbon nanofoam current collector enables a high sulfur loading of $4.8 \mathrm{mg} \mathrm{cm}^{-2}$ in the cathode, and stabilizes the high-loading sulfur cathode with a high charge storage capacity of $490-452 \mathrm{~mA} \cdot \mathrm{h} \mathrm{g}^{-1}$ for 100 continuous cycles, indicating an excellent capacity retention of $90 \%$. Inspired by these features, we further amplify the material characteristics by modifying the carbon nanofoam with graphene and molybdenum disulfide $\left(\mathrm{MoS}_{2}\right)$ coatings to boost the charge transfer and polysulfide-trapping capabilities, respectively [17-19,30-32]. The modified carbon nanofoam enables the high-loading sulfur cathode to attain a high charge- storage capacity of $672 \mathrm{~mA} \cdot \mathrm{h} \mathrm{g}^{-1}$ at the $\mathrm{C} / 10$ rate in the graphene-coated carbon nanofoam. The cathode simultaneously achieves high electrochemical utilization and stability in the $\mathrm{MoS}_{2}$-coated carbon nanofoam at a low electrolyte-to-sulfur ratio of $10 \mu \mathrm{L} \mathrm{mg}^{-1}$. Thus, in this study, we successfully demonstrate a cell configuration modification with a carbon nanofoam current collector and optimize it with a functional coating.

\section{Materials and Methods}

\subsection{Materials and Chemical Characterization}

Carbon nanofoam was obtained as commercial carbon paper (High Tech Material Solutions). The graphene-coated and $\mathrm{MoS}_{2}$-coated carbon nanofoams were prepared by chemical vapor deposition, using carbon nanofoam as the substrate. The graphene-coated carbon nanofoam was heated under argon gas at $1500 \mathrm{sccm}$, hydrogen gas at $200 \mathrm{sccm}$, and methane $\left(\mathrm{CH}_{4}\right)$ at $5 \mathrm{sccm}$ at $900{ }^{\circ} \mathrm{C}$ for $150 \mathrm{~min}$. The $\mathrm{MoS}_{2}$-coated carbon nanofoam was prepared by depositing a thin film of molybdic acid $\left(\mathrm{MoO}_{3}\right)$ on the carbon nanofoam at $300{ }^{\circ} \mathrm{C}$ and $2 \times 10^{-5}$ torr with an e-beam at $0.5 \mathrm{~A} \mathrm{~s}^{-1}$. Subsequently, the $\mathrm{MoO}_{3}$-coated carbon nanofoam was treated under hydrogen sulfide $\left(\mathrm{H}_{2} \mathrm{~S}\right)$ at 150 torr and $700{ }^{\circ} \mathrm{C}$ for an additional $60 \mathrm{~min}$. The morphology, microstructure, and elemental analyses of the various carbon nanofoams were performed under a field emission scanning electron microscope (SEM) (SU-8000, Hitachi, Tokyo, Japan) and an energy-dispersive X-ray spectroscopy (EDX) spectrometer (XFlash 5010, Bruker, Billerica, MA, USA). The porosity analysis was performed by nitrogen adsorption-desorption isotherms at $-196{ }^{\circ} \mathrm{C}$ using an automated gas sorption instrument (Autosorb $\mathrm{iQ} \mathrm{MP/MP,} \mathrm{Anton} \mathrm{Paar,} \mathrm{Austria).} \mathrm{The} \mathrm{specific} \mathrm{surface}$ area and pore volume and size were calculated by the Brunauer-Emmett-Teller (BET) 
method with a 7-point model and subsequently confirmed by the Barrett-Joyner-Halenda (BJH), density functional theory (DFT), and Horvath-Kawazoe (HK) methods. Raman microscopy was performed using a Micro-Raman and Micro-PL spectrometer (Labram HR, Jobin Yvon, Paris, France) at $514 \mathrm{~nm}$ laser excitation.

\subsection{Electrochemical and Cell Performance Characterization}

The carbon nanofoam and its two derivatives were used as porous current collectors to develop high-loading sulfur cathodes. The sulfur cathode was prepared by adding $25 \mu \mathrm{L}$ of $1.0 \mathrm{M}$ lithium polysulfide $\left(\mathrm{Li}_{2} \mathrm{~S}_{6}\right)$ catholyte into the carbon nanofoam current collector at a fixed sulfur loading of $4.8 \mathrm{mg} \mathrm{cm}^{-2}$. The $\mathrm{Li}_{2} \mathrm{~S}_{6}$ catholyte was prepared by mixing stoichiometric amounts of sulfur (99.5\%; Alfa Aesar, MA, USA) and lithium sulfide ( $\mathrm{Li}_{2} \mathrm{~S}$; $99.9 \%$; Alfa Aesar, MA, USA) with $1.85 \mathrm{M}$ bis(trifluoromethane)sulfonimide lithium salt (99.95\%; Sigma-Aldrich Corporation, MO, USA) and 0.1 M lithium nitrate co-salt (99.98\%; Alfa Aesar, MA, USA) in a 1,3-dioxolane (99+\%; Alfa Aesar, MA, USA) and 1,2-dimethoxyethane (99+\%; Alfa Aesar, MA, USA) mixture. The high-loading sulfur cathode, polymeric separator, and lithium anode (99.9\%; Sigma-Aldrich Corporation, MO, USA) were assembled in a coin cell with a low electrolyte-to-sulfur ratio of $10 \mu \mathrm{L} \mathrm{mg}^{-1}$. The electrochemical impedance spectra and cyclic voltammograms were recorded using integrated electrochemical workstations (SP-150 and VMP-300, Biologic, France) from $1 \mathrm{MHz}$ to $100 \mathrm{mHz}$ with an alternating-current voltage amplitude of $5 \mathrm{mV}$ and set between 1.5 and $3.0 \mathrm{~V}$ at a scanning rate of $0.02 \mathrm{mV} \mathrm{s}^{-1}$, respectively. The discharge/charge voltage profiles and cyclability data were collected using a programmable battery cycler (BCS-800 series, Biologic, France) in a voltage window of $1.6-2.8 \mathrm{~V}$ at the $\mathrm{C} / 10$ rate. The current density of $C / 10$ rate was calculated based on the mass loading and the theoretical charge storage capacity of sulfur (i.e., $1 \mathrm{C}=1675 \mathrm{~mA} \mathrm{~g}^{-1}$ ).

\section{Results and Discussion}

\subsection{Material Characterization of the Carbon Nanofoams}

Figure 1 presents the microstructural inspection and porosity analysis of the unmodified, graphene-coated, and $\mathrm{MoS}_{2}$-coated carbon nanofoams. In the scanning electron microscopy (SEM) images, the unmodified carbon nanofoam appears to have a rough surface composed of nanoporous carbon clusters, with a carbon nanofiber skeleton that supports the continuous conductive network (Figure 1a). This structure enables the carbon nanofoam to host the insulating solid-state active materials and liquid-state polysulfides within the porous spaces of its conductive matrix [26-28]. The hosted active materials then possess smooth charge transfer capabilities and high material stability in the cathode $[10,13]$. The two main features of the carbon nanofoam, i.e., the conductive matrix that improves the reaction kinetics of sulfur [14-23] and the porous network that enhances the electrochemical stability of the polysulfides [14-16,20-28], are optimized by surface coating the carbon nanofoam with a layer of functional coating through chemical vapor deposition. This results in the formation of the graphene-coated and $\mathrm{MoS}_{2}$-coated carbon nanofoams that have a nanocoating attached on the carbon nanofoam substrates (Figure 1b,c).

Figure $1 \mathrm{~d}$ shows a summary of the porosity analysis of the carbon nanofoams. The adsorption-desorption isotherms indicate that the nanoporous structures of the carbon nanofoams both with and without the surface coatings are similar. The carbon nanofoams show a microporous adsorption behavior at low relative pressure and a mesoporous adsorption-desorption behavior featuring the H3 loop. The H3-type hysteresis loop is shown by sheet-like materials (i.e., carbon nanofoam) and slit-shaped porous materials (i.e., graphene and $\mathrm{MoS}_{2}$ coatings). Pore analysis by adsorption and corresponding pore size distribution analysis from 0.5 to $160 \mathrm{~nm}$ are performed using the Barrett-JoynerHalenda (BJH), density functional theory (DFT), and Horvath-Kawazoe (HK) methods. The pore analysis indicates that the nanopores of the carbon nanofoams are almost identical, which is consistent with the isotherms and clearly describes the microporous and mesoporous structures of the carbon substrate. The Brunauer-Emmett-Teller (BET) method 
is subsequently used to measure the specific surface area of the carbon nanofoams. The specific surface areas (with the total pore volume and average pore size in parentheses) of the unmodified, graphene-coated, and $\mathrm{MoS}_{2}$-coated carbon nanofoams are $145.8 \mathrm{~m}^{2} \mathrm{~g}^{-1}$ $\left(0.8 \mathrm{~cm}^{3} \mathrm{~g}^{-1}\right.$ and $\left.3.8 \mathrm{~nm}\right), 164.6 \mathrm{~m}^{2} \mathrm{~g}^{-1}\left(0.7 \mathrm{~cm}^{3} \mathrm{~g}^{-1}\right.$ and $\left.3.4 \mathrm{~nm}\right)$, and $170.9 \mathrm{~m}^{2} \mathrm{~g}^{-1}$ $\left(0.7 \mathrm{~cm}^{3} \mathrm{~g}^{-1}\right.$ and $\left.3.4 \mathrm{~nm}\right)$, respectively. The detailed analytical results indicate that after surface coating, the specific surface area increases, but the pore volume and pore size decrease. Moreover, the graphene-coated and $\mathrm{MoS}_{2}$-coated carbon nanofoams display similar nanopore characteristics. The porosity analysis of the carbon nanofoams therefore demonstrates that the appropriate modification of the carbon nanofoam could generate surface graphene and $\mathrm{MoS}_{2}$ coatings. It is possible that the coating layer covers the carbon nanofoam surface and slightly decreases the porosity. The functional coating consists of layered materials with high specific surface areas, which increases the specific surface area by modifying the matrix and incorporating the slit pores of the layered structure [14-16,23-28].

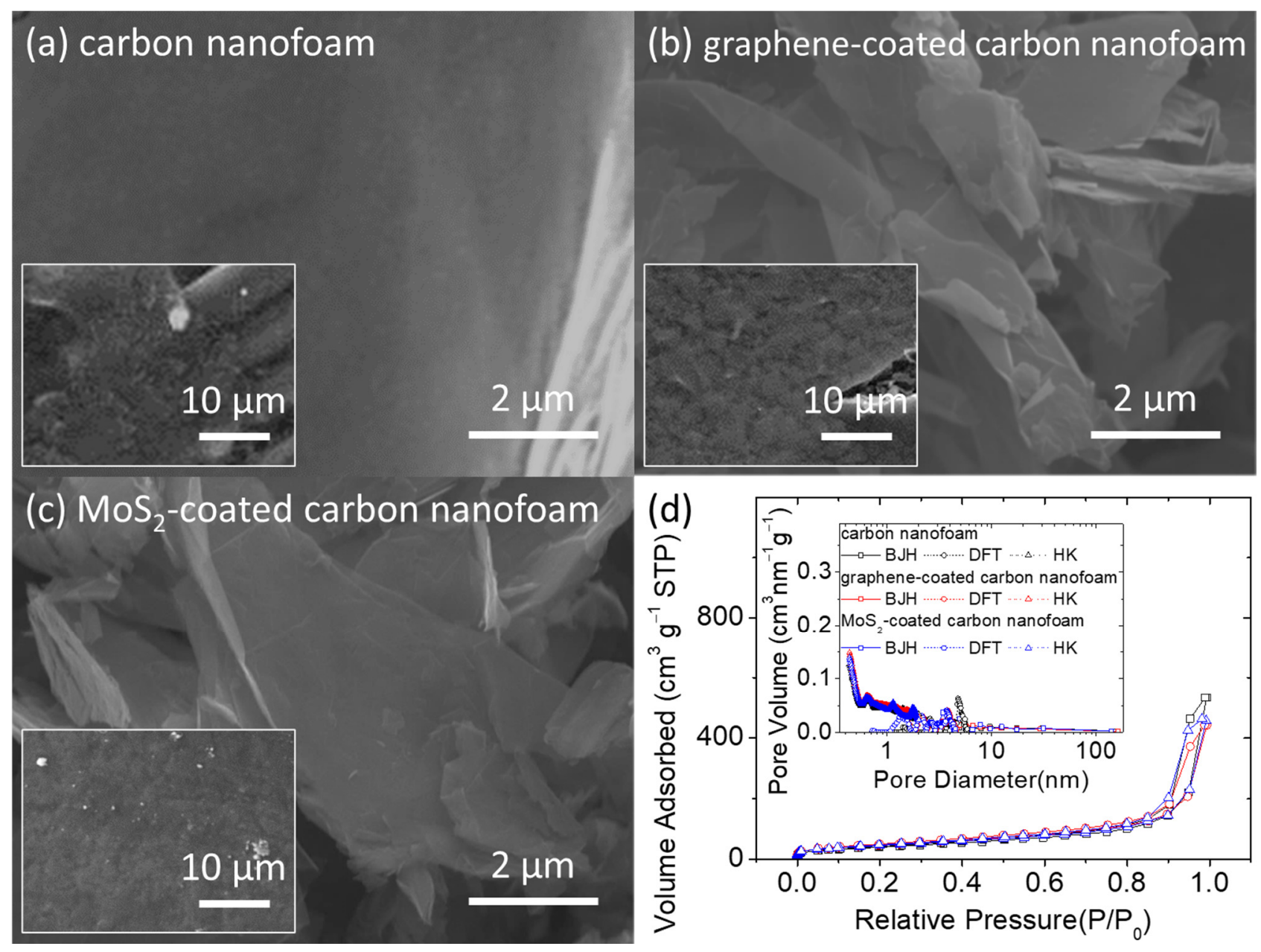

Figure 1. Material characterization: microstructural inspection of (a) carbon nanofoam, (b) graphene-coated carbon nanofoam, and (c) $\mathrm{MoS}_{2}$-coated carbon nanofoam. (d) Nitrogen adsorption-desorption isotherms; inset shows pore analysis by adsorption.

Figure 2 presents the elemental and Raman analyses of the unmodified, graphenecoated, and $\mathrm{MoS}_{2}$-coated carbon nanofoams. The energy-dispersive X-ray spectroscopy (EDX) results show high-intensity elemental carbon signals in the unmodified and graphenecoated carbon nanofoams (Figure 2a,b). The $\mathrm{MoS}_{2}$-coated carbon nanofoam shows intense elemental molybdenum and sulfur signals owing to its coating (Figure 2c). Although the $\mathrm{MoS}_{2}$-coated carbon nanofoam has a different surface elemental composition, the top-view 
microstructural observation confirms the lack of significant physical changes in, or damage to, the samples.

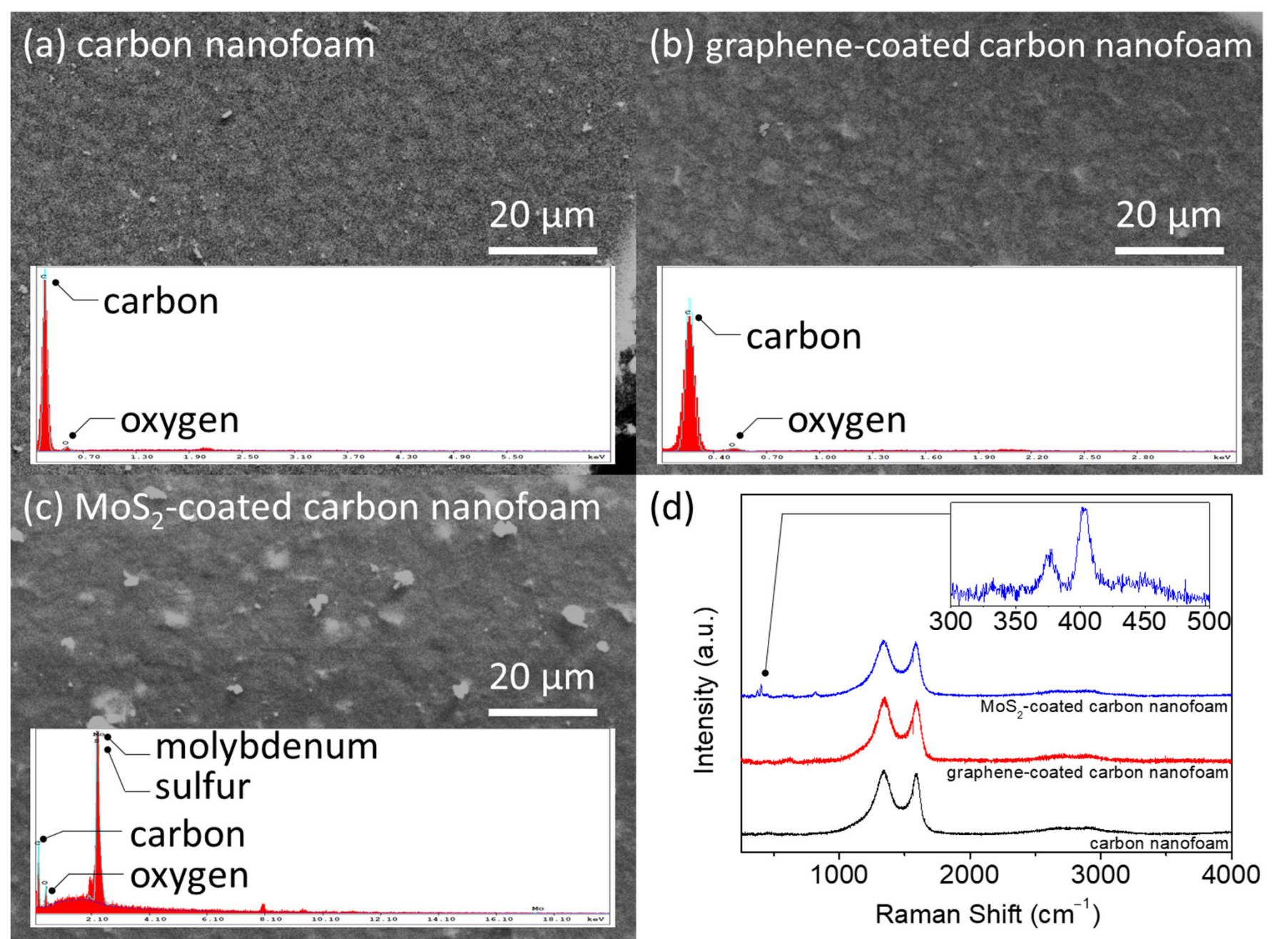

Figure 2. Chemical analysis: microstructural and elemental analyses of (a) carbon nanofoam, (b) graphene-coated carbon nanofoam, and (c) $\mathrm{MoS}_{2}$-coated carbon nanofoam. (d) Raman spectra.

Figure $2 \mathrm{~d}$ shows the Raman spectra of the carbon nanofoams. The use of a carbon nanofoam as the substrate yields a strong $\mathrm{D}$ band at $1350 \mathrm{~cm}^{-1}$ and $\mathrm{G}$ band at $1580 \mathrm{~cm}^{-1}$, which reflect disorder in the $\mathrm{sp}^{2}$-hybridized carbon systems and stretching of the C-C bond in ordered graphitic materials, respectively. Another strong peak in the $2500-2800 \mathrm{~cm}^{-1}$ range corresponds to the graphitic $\mathrm{sp}^{2}$ material $2 \mathrm{D}$ band that results from inelastic scattering due to the graphene structure [17-19]. The $\mathrm{MoS}_{2}$-coated carbon nanofoam is characterized by additional characteristic peaks $E^{1}{ }_{2 \mathrm{~g}}$ and $\mathrm{A}_{1 \mathrm{~g}}$ at $376 \mathrm{~cm}^{-1}$ and $403 \mathrm{~cm}^{-1}$, respectively, as shown in the inset. The in-plane $\mathrm{E}^{1}{ }_{2 \mathrm{~g}}$ mode results from the vibration of sulfur atoms in one direction and of the molybdenum atom in the opposite direction, and the out-of-plane $\mathrm{A}_{1 \mathrm{~g}}$ mode results from the out-of-plane vibration of the sulfur atoms [29-31]. We next examine the $\mathrm{I}_{\mathrm{D}} / \mathrm{I}_{\mathrm{G}}$ ratios of the unmodified, graphene-coated, and $\mathrm{MoS}_{2}$-coated carbon nanofoams, which are $1.66,1.84$, and 1.60 , respectively. The relatively high ratio of the graphene-coated carbon nanofoam indicates the deposition of a layer of defective graphene on the carbon nanofoam.

Based on the material characterization summarized above, it is worth noting that the unique material properties of fast electron transfer and polysulfide adsorption of the carbon nanofoam, with its conductive and porous structure, are strengthened by the graphene coating and $\mathrm{MoS}_{2}$ coating, respectively. The prominent differences in porosity 
and conductivity between the unmodified and coated nanofoams make them suitable to be used as platforms for the analysis of the battery chemistry of lithium-sulfur systems.

\subsection{Electrochemistry of the Sulfur Cathode with the Carbon Nanofoams}

Figure 3 shows a summary of the electrochemical analysis of the lithium-sulfur battery cathode with the different carbon nanofoams as advanced current collectors. The cathode electrochemistry is analyzed with a high-loading sulfur cathode that attains a sulfur loading of $4.8 \mathrm{mg} \mathrm{cm}^{-2}$ at a low electrolyte-to-sulfur ratio of $10 \mu \mathrm{L} \mathrm{mg}^{-1}$. This allows the investigation of the insulating nature of the solid-state active materials and the diffusion of the liquid-state active material, which often affect the electrochemical performance of the high-loading sulfur cathode [10-13].

(a)

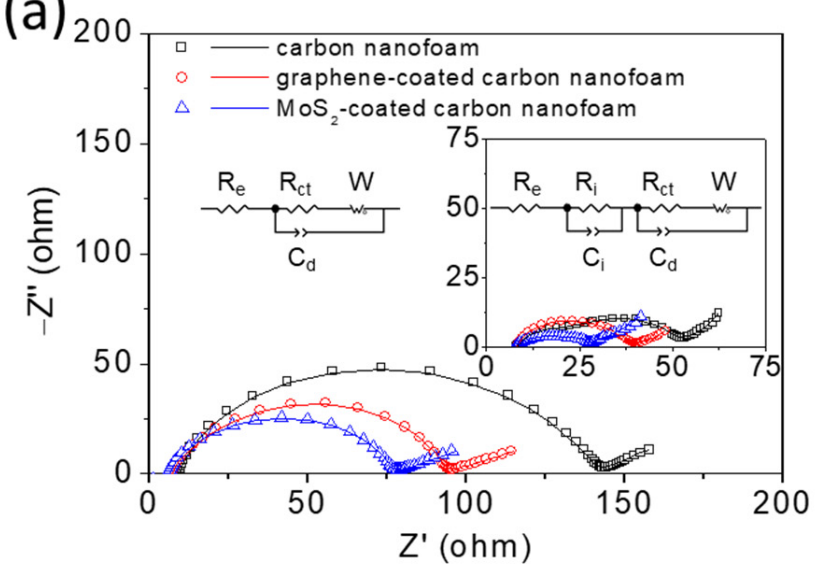

(c)

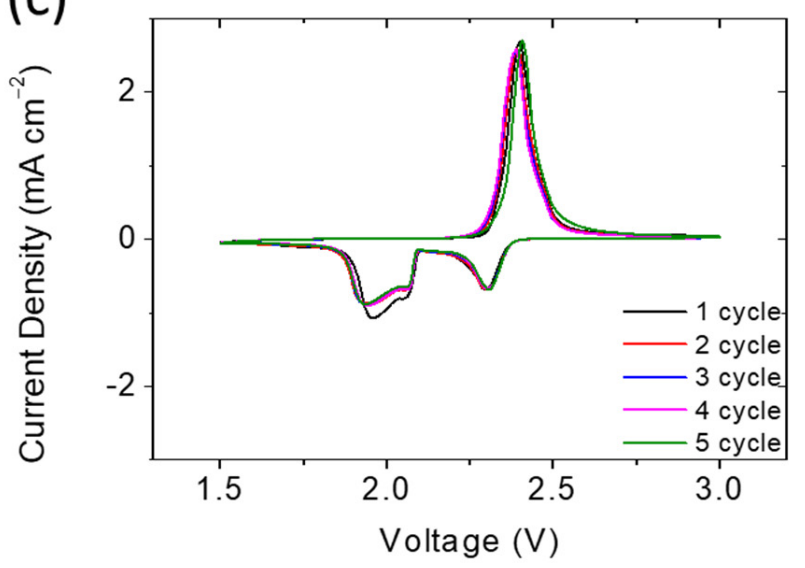

(b)

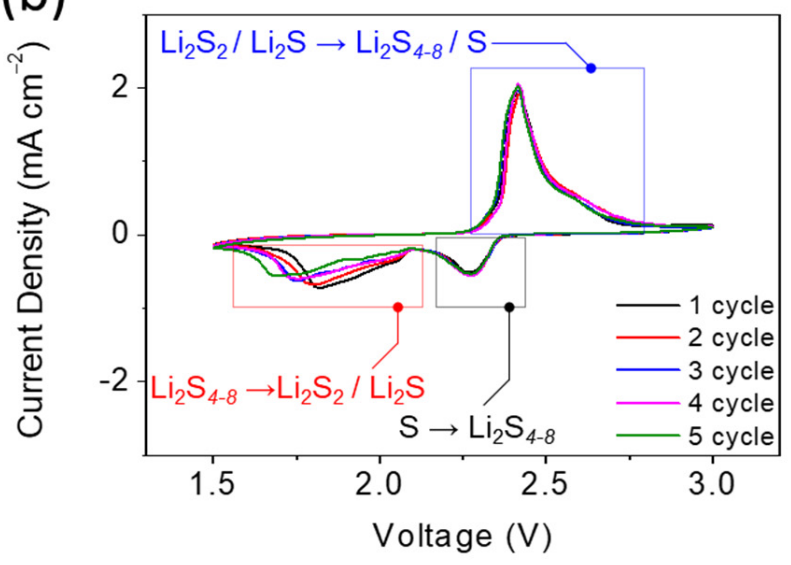

(d)

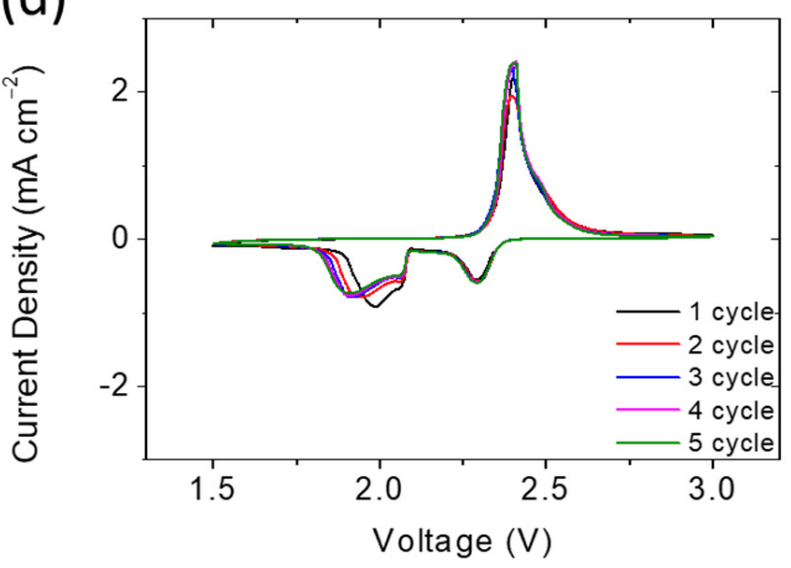

Figure 3. Electrochemical analysis: (a) electrochemical impedance analysis of the high-loading sulfur cathodes with various carbon nanofoams before and after cycling (inset). The data points are the experimental impedance data, and the data curves are the fitting impedance data obtained by the equivalent circuits. Cyclic voltammograms of sulfur cathodes with (b) the carbon nanofoam, (c) the graphene-coated carbon nanofoam, and (d) the $\mathrm{MoS}_{2}$-coated carbon nanofoam at $0.02 \mathrm{mV} \mathrm{s}^{-1}$.

Figure 3a presents the electrochemical impedance analysis of cells with different carbon nanofoams to explore the impedance/resistance before and after cycling (the inset). The Nyquist plots (i.e., the data points) with the corresponding equivalent circuits (i.e., the fitting curves) indicate a semicircle and a slash before cycling, which are associated with the ohmic resistance ( $\mathrm{Re}$ ) of the whole device, the charge transfer resistance (Rct) of the charge transfer kinetics, and Warburg impendence $(W)$ of the lithium-ion diffusion, respectively [10-13]. The high-loading sulfur cathodes with the unmodified, graphene-coated, 
and $\mathrm{MoS}_{2}$-coated carbon nanofoams possess the Re and Rct values of 9.3 and 133.9, 7.9 and 87.4, and 7.7 and $71.3 \Omega$, respectively (Table S1, Supplementary Materials). The highloading sulfur cathode with the carbon nanofoam has a low charge transfer resistance because the carbon nanofoam accommodates the large amount of active material within its conductive carbon matrix. The graphene and $\mathrm{MoS}_{2}$ coatings further reduce the resistance because the graphene coating improves the charge transfer of the cathode [10,17-19] and the $\mathrm{MoS}_{2}$ coating confers both high electron mobility and polysulfide stabilization [10,30-32]. These features result in low resistance, which could improve the reaction kinetics and electrochemical efficiency of the cathode. The inset shows the electrochemical impedance analysis of the cells after cycling, which depicts the interface resistance (Ri) that corresponds to the deposition of a solid insulation layer on the surface of the electrodes at the high frequency region [10-13]. The cycled sulfur cathodes with the unmodified, graphene-coated, and $\mathrm{MoS}_{2}$-coated carbon nanofoams possess the Re, Ri, and Rct values of 8.1, 9.3, and 36.6, 7.8, 8.9, and 23.3, and 7.6, 9.1, and $11.3 \Omega$, respectively (Table S1, Supplementary Materials). As a reference, the interface capacitance $(\mathrm{Ci})$ and the double-layer capacitance $(\mathrm{Cd})$ are the constant phase elements. The impedance semicircles of the cathode with different carbon nanofoams decrease because the carbon nanofoams serve as a conductive network, trap the active material, and reactivate the trapped active material during cycling. This limits the formation of insulating active material agglomerates while retaining the dissolved polysulfides within the cathode region as the catholyte [12,26-28]. The graphene-coated and $\mathrm{MoS}_{2}$-coated carbon nanofoams exhibit even lower cathode resistance as the coatings confer high conductance and polysulfide trapping, respectively. Thus, the electrochemical impedance results demonstrate that the carbon nanofoam current collectors endow the high-loading sulfur cathode with low cathode resistance and high utilization of the trapped active material.

Figure $3 \mathrm{~b}-\mathrm{d}$ show the cyclic voltammograms of the unmodified, graphene-coated, and $\mathrm{MoS}_{2}$-coated carbon nanofoams, respectively. The cyclic voltammograms of all three types of carbon nanofoams have similar shapes; each has a pair of cathodic peaks and a continuous anodic peak. The two cathodic peaks reflect the reduction conversion from solid-state sulfur to liquid-state polysulfides at $\sim 2.4 \mathrm{~V}$ (cathodic-1 peak) and then to solidstate sulfide mixtures $\left(\mathrm{Li}_{2} \mathrm{~S}_{2} / \mathrm{Li}_{2} \mathrm{~S}\right)$ at $\sim 2.1 \mathrm{~V}$ (cathodic-2 peak) [5,9-11]. The continuous anodic peaks at $\sim 2.3 \mathrm{~V}$ (anodic peak) correspond to the reversible oxidation from solid-state sulfides to liquid-state polysulfides and solid-state sulfur [5,9-11]. The high-loading sulfur cathode with the carbon nanofoam shows almost unchanged cathodic- 1 and anodic peaks, indicating the excellent polysulfide retention and enhanced redox reaction. However, the overlapping cathodic-2 peak shows a slight shift of the peak current density toward the low voltage region, which implies a slight increase in the polarization of the high-loading sulfur cathode in the lean-electrolyte lithium-sulfur batteries; however, the slight increase in the polarization would not affect the electrochemical reversibility of the cell. On the other hand, notably, repeated scans of the graphene-coated and $\mathrm{MoS}_{2}$-coated carbon nanofoams do not reveal any apparent decreases in current or shifts in potential in these reduction and oxidation peaks. The overlapping redox curves attest to the superior cell reversibility and stability contributed by the coated nanofoams [9-11]. The improved electrochemical reaction of the graphene-coated carbon nanofoam mainly arises from the conductive graphene coating, which amplifies the fast charge transfer of the matrix. Thus, the sluggish reduction reaction from polysulfide to sulfide is ameliorated, which maintain the reduction peaks as almost unchanged; the solid-state active material maintains the high reutilization during cycling [10,17-19]. The enhanced redox reaction of the $\mathrm{MoS}_{2}$-coated carbon nanofoam could be attributed to the $\mathrm{MoS}_{2}$ coating, which confers a strong polysulfidetrapping capability and fast electron mobility. The resulting strong polysulfide retention and high reaction kinetics are characterized by the overlapping reduction and oxidation peaks [10,30-32]. Thus, electrochemical analysis of the carbon nanofoams indicates that the use of the carbon nanofoam as a porous current collector would allow the development of a high-performance sulfur cathode with a high sulfur loading of $4.8 \mathrm{mg} \mathrm{cm}^{-2}$ and high 
electrochemical reversibility at the low electrolyte-to-sulfur ratio of $10 \mu \mathrm{L} \mathrm{mg}^{-1}$. Moreover, optimally coated carbon nanofoams could boost the electrochemical performance with high utilization and stability.

Figure $4 \mathrm{a}-\mathrm{c}$ present the discharge/charge voltage profiles of cells with different carbon nanofoams over 100 cycles. The cell with the unmodified carbon nanofoam (Figure 4a) displays two distinguishable discharge plateaus and a continuous charge plateau, consistent with the cyclic voltammograms. During discharge, the two separate discharge plateaus indicate the two reduction reactions: the reduction from sulfur to polysulfides at $\sim 2.4 \mathrm{~V}$ and the subsequent reduction from polysulfides to sulfides at $\sim 2.1 \mathrm{~V}[5,9-11]$. The overlapping upper discharge curves indicate the advantages of using the carbon nanofoam to host the large amount of active material and inhibit the fast polysulfide diffusion. The lower discharge plateaus are well-retained during cycling, representing the improved reaction kinetics of the cathode that has a large amount of insulating sulfur and a low amount of electrolyte. Moreover, the carbon nanofoam continuously transfers electrons and channels electrolyte to reactivate the trapped active material, facilitating the stable cell cycling with high retention. During charging, the two continuous plateaus at $\sim 2.3 \mathrm{~V}$ could be attributed to the reversible oxidation reactions of sulfide to polysulfides and sulfur [9-11]. Figure $4 b, c$ show the discharge/charge voltage profiles of cells with the graphene-coated and $\mathrm{MoS}_{2-}$ coated carbon nanofoams, respectively. The high-conductivity graphene coating confers fast charge transfer capabilities to the active material in the cathode, which is trapped in the carbon nanofoam. This facilitates the electrochemical utilization of sulfur (Figure $4 \mathrm{~b}$ ). In addition to enhanced conductivity, the $\mathrm{MoS}_{2}$-coated carbon nanofoam allows the original carbon nanofoam to physically trap the diffusing polysulfides and chemically adsorb them, further improving the electrochemical stability during cell discharge and charge (Figure 4c).

(a)

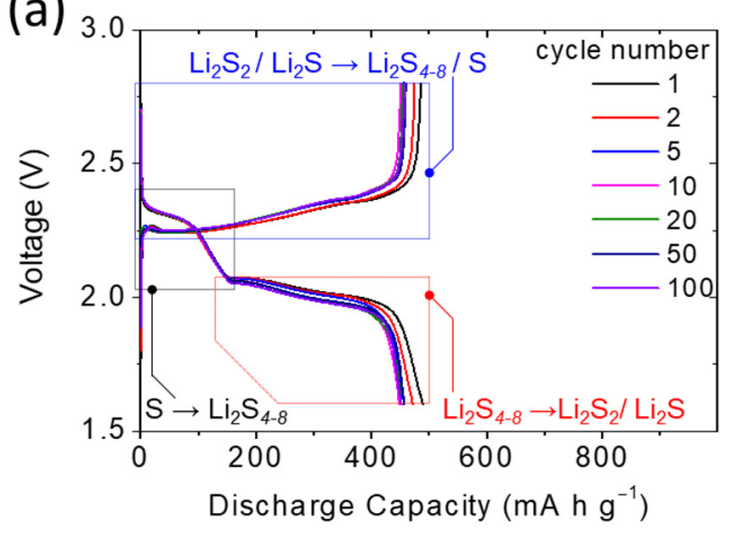

(c)

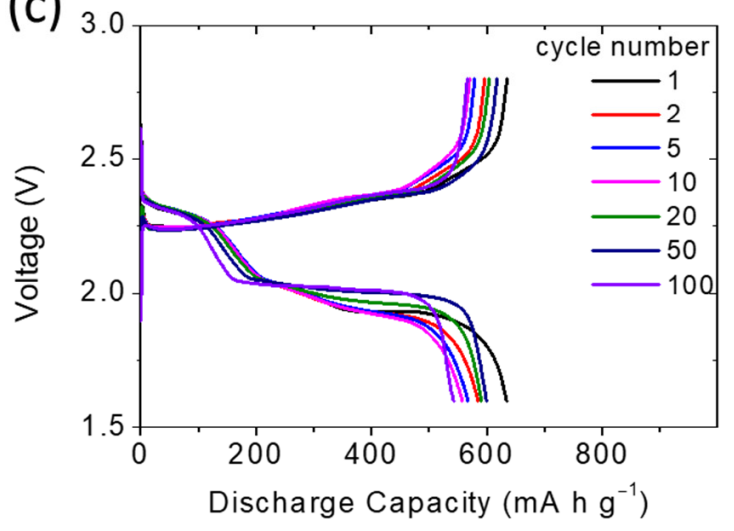

(b)

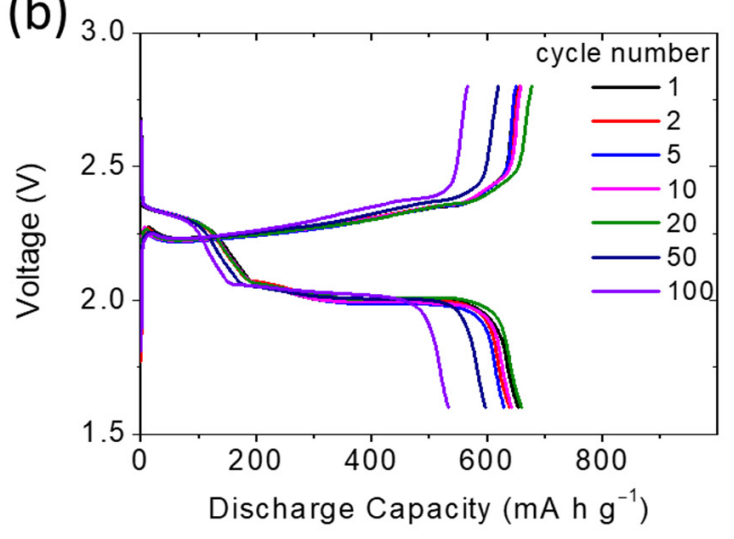

(d)

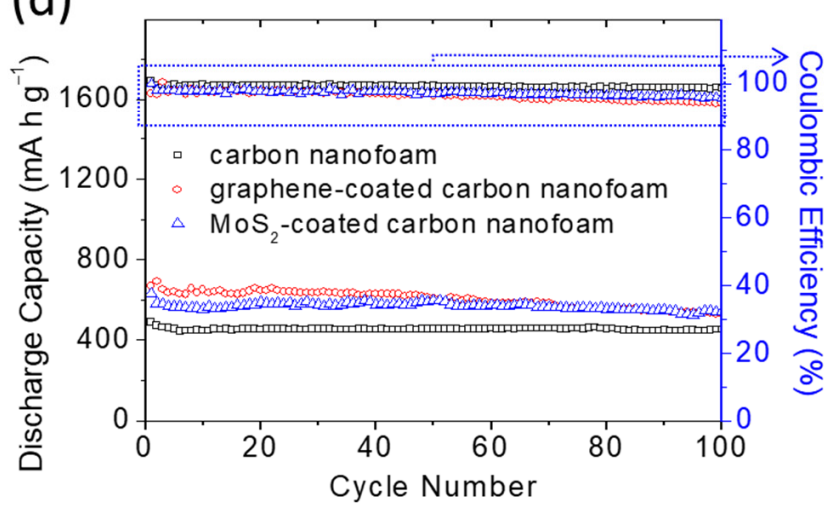

Figure 4. Electrochemical performance: discharge/charge voltage profiles of sulfur cathodes with (a) the carbon nanofoam, (b) the graphene-coated carbon nanofoam, and (c) the $\mathrm{MoS}_{2}$-coated carbon nanofoam. (d) Cyclability at the C/10 rate. 
Figure $4 \mathrm{~d}$ shows the cyclability of the high-loading sulfur cathode with the carbon nanofoam current collectors. The cycling performance demonstrates that the carbon nanofoam current collector significantly enhances the electrochemical stability and efficiency of the high-loading sulfur cathode in the lean-electrolyte lithium-sulfur cell. The graphene-coated and $\mathrm{MoS}_{2}$-coated carbon nanofoams further improve the electrochemical utilization of the large amount of sulfur. The high-loading sulfur cathodes with the unmodified, graphene-coated, and $\mathrm{MoS}_{2}$-coated carbon nanofoams attain high charge storage capacities of 490,672 , and $633 \mathrm{~mA} \cdot \mathrm{h} \mathrm{g}^{-1}$, respectively, at the C/10 rate. After 100 cycles, the reversible capacities remain as high as 452,532 , and $548 \mathrm{~mA} \cdot \mathrm{h} \mathrm{g}^{-1}$ at the $\mathrm{C} / 10$ rate, respectively, corresponding to high capacity retentions of 92,79 , and $87 \%$, respectively. This superior cycling stability suggests that the carbon nanofoam current collectors provide a more stable electrochemical environment for the electrochemical conversion-type sulfur cathode than conventional cells, which are optimal cathode configuration for high-loading sulfur cathodes.

After the electrochemical analysis, we retrieved the high-loading sulfur cathodes from the cycled cells after 100 cycles at discharge status. Figure 5a-c show the SEM inspection of the carbon nanofoams of the cycled cathodes. The microstructural images depict the distinguishable retention of the active material, with no obvious agglomeration of the insulating solid-state active materials after cycling. This positive feature confirms the good encapsulation and stabilization of the active material in the cathode [26-28]. Moreover, the battery performance depicts the research trend in developing high-loading sulfur cathodes in the lean-electrolyte lithium-sulfur cells with enhanced electrochemical stability (Figure $5 \mathrm{~d}$ and Table S2, Supplementary Materials). The analytical results indicate the necessity in designing and analyzing the high-loading sulfur cathode in the cell with low amounts of electrolyte featuring a low electrolyte-to-sulfur ratio while maintaining the necessary cycle stability for realizing long cycle life with high capacity retention [10-16]. Among the published lithium-sulfur studies that report on the sulfur loading and electrolyte-tosulfur ratio, our carbon nanofoam simultaneously attains high sulfur loading and a low electrolyte-to-sulfur ratio while attaining the necessary cycle life featuring the highest capacity retention. Therefore, the comparison analysis confirms the feasibility of adopting the carbon nanofoams in designing high-sulfur-loading cathodes with high electrochemical performance at a low electrolyte condition.

The high electrochemical stability of the carbon nanofoams confirms that the inclusion of conductive and porous current collectors improves the cycle stability by hosting the insulating active material in the cathode substrate and stabilizing the diffusing polysulfides within the cathode region. The conductive skeleton and porous network of the carbon nanofoam confer the trapped active material with fast electron transfer and smooth electrolyte diffusion capabilities, resulting in superior retention of the active material and higher capacity [12-14,26-28]. The graphene-coated carbon nanofoam attains the highest peak charge storage capacity because the conductive graphene coating improves the conductivity of the cathode, which further improves the reaction kinetics and electrochemical utilization [17-19]. The $\mathrm{MoS}_{2}$-coated carbon nanofoam also shows good conductivity and further possesses a strong polysulfide-trapping capability owing to the $\mathrm{MoS}_{2}$ coating, which improves the electrochemical utilization and stability of the high-loading sulfur cathode [30-32]. 


\section{(a) carbon nanofoam}

(b) graphene-coated carbon nanofoam
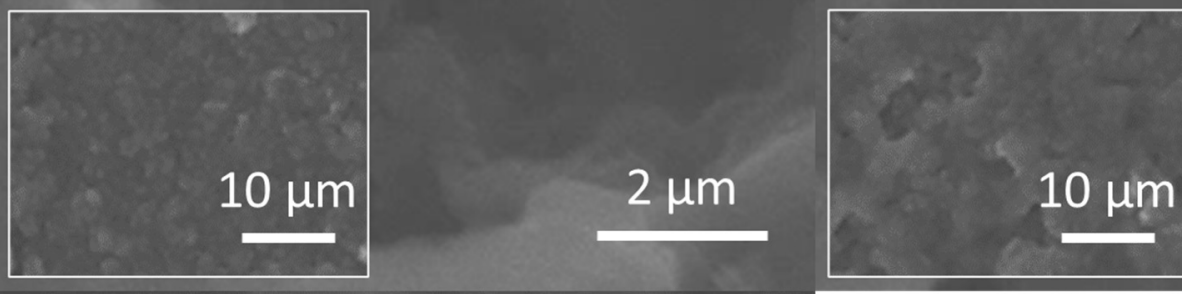

(c) $\mathrm{MoS}_{2}$-coated carbon nanofoam

(d)
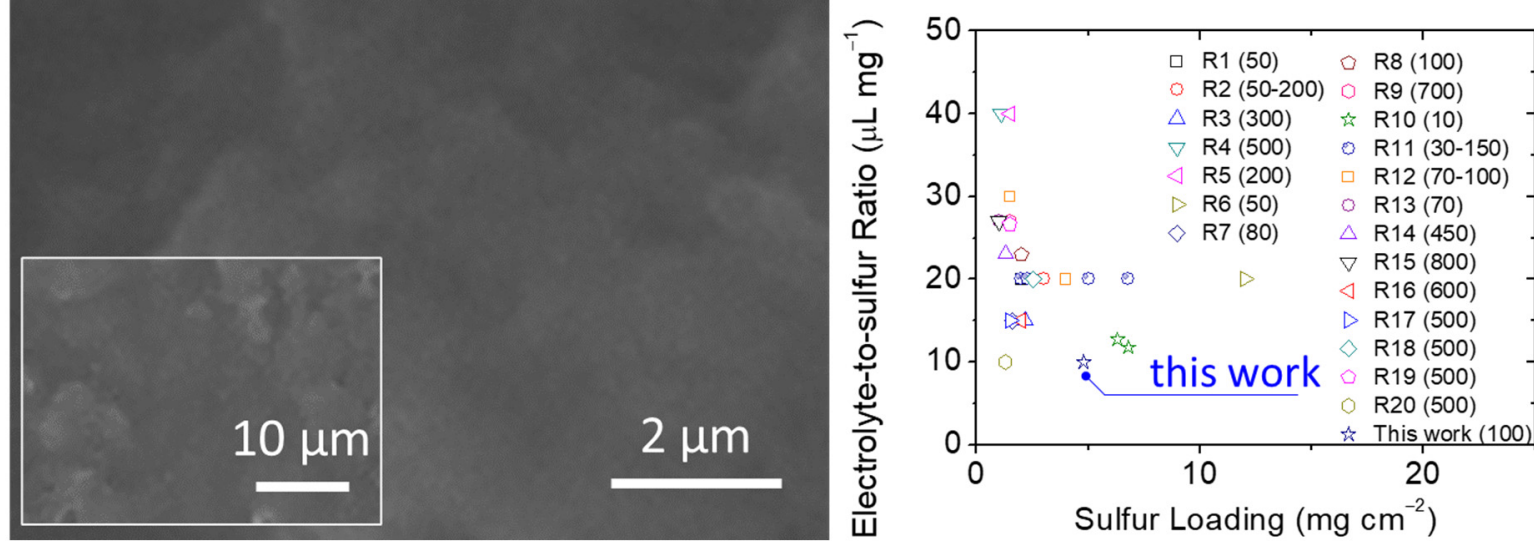

Figure 5. Battery performance: microstructural inspection of (a) carbon nanofoam, (b) graphene-coated carbon nanofoam, and (c) $\mathrm{MoS}_{2}$-coated carbon nanofoam retrieved from cycled cathodes after 100 cycles. (d) Comparison with literature data: sulfur loading, electrolyte-to-sulfur ratio, and the cycle life in the parentheses.

\section{Conclusions}

In summary, the carbon nanofoam current collector offers a practical option for the development of a high-performance sulfur cathode with high sulfur loading and enhanced electrochemical stability in a lean-electrolyte lithium-sulfur cell. The improvements in the overall cathode parameters and performance arise from the conductive and porous structure of the carbon nanofoam, which hastens the sluggish conversion reaction between the solid-state and liquid-state active materials, encapsulates a large amount of sulfur, and traps the migrating polysulfides within the cathode as catholytes. The modified graphene-coated and $\mathrm{MoS}_{2}$-coated carbon nanofoams further amplify the desirable material properties of carbon nanofoams by virtue of the conductive graphene and polysulfidetrapping $\mathrm{MoS}_{2}$ coatings, respectively. The high-loading sulfur cathodes with graphenecoated and $\mathrm{MoS}_{2}$-coated carbon nanofoams exhibit high charge -storage capacities of 672 and $633 \mathrm{~mA} \cdot \mathrm{h} \mathrm{g}^{-1}$, respectively, with a superior cycle stability and high capacity retention of $79-87 \%$ after 100 cycles.

Supplementary Materials: The following are available online at https:/ /www.mdpi.com/article/10 .3390 / nano11082083/s1, Table S1: Electrochemical impedance analysis of the high-loading sulfur cathodes with various carbon nanofoams before and after cycling, Table S2: Comparative analysis of the battery performances and electrochemical characteristics of the sulfur cathodes in the lithiumsulfur research, supporting references: R1-R20. 
Author Contributions: Conceptualization and methodology: S.-Y.C. and S.-H.C.; writing-original draft preparation and writing-review and editing: S.-H.C. All authors have read and agreed to the published version of the manuscript.

Funding: This research was funded by the Ministry of Education in Taiwan under the Yushan Young Scholar Program and the Ministry of Science and Technology in Taiwan under grant MOST 110-2636E-006-012 (Young Scholar Fellowship Program), grant MOST 109-2923-E-006-006, and grant MOST 110-2623-E-006-002.

Acknowledgments: This research was supported in part by the Higher Education Sprout Project, Ministry of Education to the Headquarters of University Advancement at National Cheng Kung University (NCKU). The authors gratefully acknowledge the use of EM003600, 2108, 2109, and 4201 of MOST 110-2731-M-006-001 belonging to the Core Facility Center of National Cheng Kung University.

Conflicts of Interest: The authors declare no conflict of interest.

\section{References}

1. Li, M.; Lu, J.; Chen, Z.; Amine, K. 30 years of lithium-ion batteries. Adv. Mater. 2018, 30, 1800561. [CrossRef] [PubMed]

2. Goodenough, J.B.; Park, K.-S. The Li-ion rechargeable battery: A perspective. J. Am. Chem. Soc. 2013, 135, 1167-1176. [CrossRef]

3. Manthiram, A. A reflection on lithium-ion battery cathode chemistry. Nat. Commun. 2020, 11, 1550. [CrossRef]

4. Manthiram, A.; Goodenough, J.B. Layered lithium cobalt oxide cathodes. Nat. Energy 2021, 6, 323. [CrossRef]

5. Chung, S.-H.; Manthiram, A. Current status and future prospects of metal-sulfur batteries. Adv. Mater. 2019, $30,1901125$. [CrossRef]

6. Li, T.; Bai, X.; Gulzar, U.; Bai, Y.-J.; Capiglia, C.; Deng, W.; Zhou, X.; Liu, Z.; Feng, Z.; Zaccaria, R.P. A comprehensive understanding of lithium-sulfur battery technology. Adv. Funct. Mater. 2019, 29, 1901730. [CrossRef]

7. Zhao, M.; Li, B.-Q.; Zhang, X.-Q.; Huang, J.-Q.; Zhang, Q. A Perspective toward practical lithium-sulfur batteries. ACS Cent. Sci. 2020, 6, 1095-1104. [CrossRef]

8. Yang, L.; Li, Q.; Wang, Y.; Chen, Y.; Guo, X.; Wu, Z.; Chen, G.; Zhong, B.; Xiang, W.; Zhong, Y. A review of cathode materials in lithium-sulfur batteries. Ionics 2020, 26, 5299-5318. [CrossRef]

9. Yin, Y.-X.; Xin, S.; Guo, Y.-G.; Wan, L.-J. Lithium-sulfur batteries: Electrochemistry, materials, and prospects. Angew. Chem. Int. Ed. 2013, 52, 13186-13200. [CrossRef]

10. Wild, M.; O’Neill, L.; Zhang, T.; Purkayastha, R.; Minton, G.; Marinescu, M.; Offer, G.J. Lithium sulfur batteries, a mechanistic review. Energy Environ. Sci. 2015, 8, 3477-3494. [CrossRef]

11. Yuan, H.; Peng, H.-J.; Huang, J.-Q.; Zhang, Q. Sulfur redox reactions at working interfaces in lithium-sulfur batteries: A perspective. Adv. Mater. Inter. 2019, 6, 1802046. [CrossRef]

12. Li, G.; Wang, S.; Zhang, Y.; Li, M.; Chen, Z.; Lu, J. Revisiting the role of polysulfides in lithium-sulfur batteries. Adv. Mater. 2018, 30, 1705590. [CrossRef]

13. Ho, Y.-C.; Chung, S.-H. A Design of the cathode substrate for high-loading polysulfide cathodes in lean-electrolyte lithium-sulfur cells. Chem. Eng. J. 2021, 422, 130363. [CrossRef]

14. Fang, R.; Zhao, S.; Sun, Z.; Wang, D.-W.; Cheng, H.-M.; Li, F. More reliable lithium-sulfur batteries: Status, solutions and prospects. Adv. Mater. 2017, 29, 1606823. [CrossRef] [PubMed]

15. Peng, H.-J.; Huang, J.-Q.; Cheng, X.-B.; Zhang, Q. Review on high-loading and high-energy lithium-sulfur batteries. Adv. Energy Mater. 2017, 7, 1700260. [CrossRef]

16. Zhao, M.; Li, B.-Q.; Peng, H.-J.; Yuan, H.; Wei, J.-Y.; Huang, J.-Q. Lithium-sulfur batteries under lean electrolyte conditions: Challenges and opportunities. Angew. Chem. Int. Ed. 2020, 59, 12636-12652. [CrossRef]

17. Zhang, Q.; Cheng, X.-B.; Huang, J.-Q.; Peng, H.-J.; Wei, F. Review of carbon materials for advanced lithium-sulfur batteries. Carbon 2015, 81, 850. [CrossRef]

18. Xu, Z.-L.; Kim, J.-K.; Kang, K. Carbon nanomaterials for advanced lithium sulfur batteries. Nanotoday 2018, 19, 84-107. [CrossRef]

19. Li, S.; Jin, B.; Zhai, X.; Li, H.; Jiang, Q. Review of carbon materials for lithium-sulfur batteries. ChemistrySelect 2018, 3, 2245-2260. [CrossRef]

20. Fang, R.; Chen, K.; Yin, L.; Sun, Z.; Li, F.; Cheng, H.-M. The regulating role of carbon nanotubes and graphene in lithium-ion and lithium-sulfur batteries. Adv. Mater. 2019, 31, 1800863. [CrossRef]

21. Yue, Z.; Dunya, H.; Kucuk, K.; Aryal, S.; Ma, Q.; Antonov, S.; Ashuri, M.; Alabbad, B.; Lin, Y.; Segre, C.U.; et al. MnO ${ }_{2-}$ coated sulfur-filled hollow carbon nanosphere-based cathode materials for enhancing electrochemical performance of Li-S cells. J. Electrochem. Soc. 2019, 166, A1355-A1362. [CrossRef]

22. Dunya, H.; Ashuri, M.; Alramahi, D.; Yue, Z.; Kucuk, K.; Segre, C.U.; Mandal, B.J. $\mathrm{MnO}_{2}$-coated dual core-shell spindle-like nanorods for improved capacity retention of lithium-sulfur batteries. ChemEngineering 2020, 4, 42. [CrossRef]

23. Dunya, H.; Ashuri, M.; Yue, Z.; Kucuk, K.; Lin, Y.; Alramahi, D.; Segre, G.U.; Mandal, B.K. Rational design of titanium oxidecoated dual Core-Shell sulfur nanocomposite cathode for highly stable lithium-sulfur batteries. J. Phys. Chem. Solids 2021, 149, 109791. [CrossRef] 
24. Wang, M.; Xia, X.; Zhong, Y.; Wu, J.; Xu, R.; Yao, Z.; Wang, D.; Tang, W.; Wang, X.; Tu, J. Porous carbon hosts for lithium-sulfur batteries. Chem. Eur. J. 2019, 25, 3710-3725. [CrossRef]

25. Zhao, M.; Peng, H.-J.; Li, B.-Q.; Chen, X.; Xie, J.; Liu, X.; Zhang, Q.; Huang, J.-Q. Electrochemical phase evolution of metal-based pre-catalysts for high-rate polysulfide conversion. Angew. Chem. Int. Ed. 2020, 132, 9096-9102. [CrossRef]

26. Fu, Y.; Su, Y.-S.; Manthiram, A. Highly reversible lithium/dissolved polysulfide batteries with carbon nanotube electrodes. Angew. Chem. Int. Ed. 2013, 52, 6930-6935. [CrossRef]

27. Yen, Y.-J.; Chung, S.-H. Lean-electrolyte lithium-sulfur electrochemical cells with high-loading carbon nanotube/nanofiberpolysulfide cathodes. Chem. Commun. 2021, 57, 2009-2012. [CrossRef]

28. Chiu, L.-L.; Chung, S.-H. A poly (ethylene oxide)/lithium bis (trifluoromethanesulfonyl) imide-coated polypropylene membrane for a high-loading lithium-sulfur battery. Polymers 2021, 13, 535. [CrossRef]

29. Zhu, P.; Gastol, D.; Marshall, J.; Sommerville, R.; Goodship, V.; Kendrick, E. A review of current collectors for lithium-ion batteries. J. Power Sources 2021, 485, 229321. [CrossRef]

30. Tian, C.; Wu, J.; Ma, Z.; Li, B.; Zhang, X.; Zu, X.; Xiang, X.; Li, S. A melt-diffusion strategy for tunable sulfur loading on CC@MoS 2 for lithium-sulfur batteries. Energy Rep. 2020, 6, 172-180. [CrossRef]

31. Xu, Z.-L.; Onofrio, N.; Wang, J. Boosting the anchoring and catalytic capability of $\mathrm{MoS}_{2}$ for high-loading lithium sulfur batteries. J. Mater. Chem. A 2020, 8, 17646-17656. [CrossRef]

32. Wang, M.; Yang, H.; Shen, K.; Xu, H.; Wang, W.; Yang, Z.; Zhang, L.; Chen, J.; Huang, Y.; Chen, M.; et al. Stable lithium sulfur battery based on in situ electrocatalytically formed $\mathrm{Li}_{2} \mathrm{~S}$ on metallic $\mathrm{MoS}_{2}$-carbon cloth support. Small Method 2020, $4,2000353$. [CrossRef] 